\title{
Qualitätsarbeit auch auf schwierig messbarem Terrain
}

Eine in der Schweiz selbstverständliche qualitätssichernde Massnahme ist, den Strassenverkehr in Tunnels mit Kameras zu überwachen. Im Bedarfsfall kann so für Regulierungs- oder Rettungsmassnahmen schneller eingegriffen werden. Auch in einem schwierig messbaren Bereich wie der Psychotherapie kann über ein LiveMonitoring die Qualität gesichert werden.

Sebastian Pfaundler

Korrespondenz:

Dr. med. Sebastian Pfaundler Psychiatrie und Psychotherapie FMH

Fläscherstrasse 37

CH-7310 Bad Ragaz

Tel. 0813027771

s.pfaundler@psyqual.ch

\section{Standard in der Chirurgie}

Qualitätsmonitoring in der Medizin ist ein aktuelles und viel diskutiertes Thema $[1,2]$.

In der Chirurgie ist es seit jeher üblich, dass beim Operieren neben der Indikationsstellung und dem eigentlichen «Handwerk» auch zahlreiche flankierende, qualitätssichernde Massnahmen ergriffen werden, vorrangig sind hierbei Sorgfalt und Sterilität. Zudem hat der Chirurg während des Eingriffs in der Regel mindestens eine Operationsschwester und einen Assistenten zur Seite. Vier beziehungsweise sechs Augen und Hände sehen, prüfen und können mehr für Qualität ausrichten als nur ein Augen- und Händepaar.

Bisher nicht so in der «Seelenchirurgie», der Psychotherapie. In der Regel sind dort Psychiater und Patient alleine bei der Arbeit. Retrospektive Betrachtungen und Analysen sind allerdings schon jetzt Bestandteil von Supervisionen. Dabei werden meist Niederschriften und mündliche Kommentare des Thera- statt. Dabei bleiben Patient und Therapeut dank eines Einwegspiegels meist klar getrennt vom beobachtenden Supervisor. Noch seltener (häufig irritierend bis störend und daher nur wenig sinnvoll) nimmt ein Supervisor im selben Raum gemeinsam mit Patient und Therapeut an einer Sitzung teil. Zwar wäre auf diese Weise ein unmittelbarerer Austausch ohne lange zeitliche Verzögerung möglich, bei dringlichen Fragen aber oder wenn der Supervisor während einer Live-Sitzung intervenieren will, müsste er den Therapeut-Patienten-Dialog unterbrechen und sich vornehmlich separat mit dem Arzt besprechen. Solche Pausen erscheinen unnatürlich und können sich auf die Therapeut-Patienten-Allianz schwächend auswirken.

\section{Direkter Einblick durch Live-Monitoring}

Sinnvoll wäre ein direkter Einblick in die Qualität des aktuellen Arbeitsprozesses mit der Möglichkeit, sich störungsfrei einzuschalten. Dies ist gewährleistet,

\section{Durch Kamera und Live-Kommentierung gelingt es, an einem Therapieprozess ohne wesentliche Störung teilzunehmen}

peuten im nachhinein mit einem Supervisor durchleuchtet und aufbereitet. Eine Präzisierung stellen dabei audiovisuelle Aufzeichnungen dar, weil diese eine wesentlich minutiösere Beobachtung mit mehr Transparenz des therapeutischen Prozesses zwischen Arzt und Patient erlauben [3].

\section{Live-Supervision bisher nur ausnahmsweise}

Dass ein Supervisor live, also zeitgleich, an einer Therapiesitzung teilnimmt, findet im allgemeinen nur ausnahmsweise und meist im Ausbildungskontext wenn im Einverständnis mit Patient und Therapeut die Therapiesitzung live audiovisuell auf einen Bildschirm zum externen Supervisor übertragen - und auch auf einem Speichermedium (Videotape, DVD, Festplatte usw.) aufgezeichnet wird. Eine Innovation stellt hierbei das vom Autor entwickelte Instrument des Schrift-gestützten Live-Monitorings dar.

Der Supervisor kann seinen Kommentar hierbei schriftlich auf einem Bildschirm im Sichtfeld des Therapeuten live einbringen. Er macht dies, indem er zum Beispiel in ein Notebook schreibt mit zusätzli- 
chem externen Bildschirm, der sich vor dem Therapeuten befindet. Der Patient weiss das, sieht aber nur die Rückseite dieses Monitors. Dadurch wird er weder gestört noch beeinflusst, und der unmittelbare Austausch und Dialog zwischen Patient und Therapeut bleiben authentisch erhalten. Dies wiederum verfolgt

\section{Der Supervisor als Operations-Assistenz}

Wie der Operationssaal eine Voraussetzung für die Chirurgie ist, so ist bei der psychotherapeutischen Arbeit die klare Positionierung von ausschliesslich Patient und Therapeut in einem eigenen Raum Voraussetzung zur Sicherung vor Störeinflüssen. Die

\section{Der Patient profitiert am meisten, weil ihm in der Therapie mit Live- Monitoring kumuliertes Know-how zur Verfügung gestellt wird}

der Supervisor auf dem Monitor der audiovisuellen Live-Übertragung zeitgleich mit.

Unabdingbar für beide Seiten ist hierbei das Vertrauen, dass es sich ausschliesslich um eine Massnahme zur Sicherung der Qualität handelt und nicht um eine Sanktionierungsvorgabe. Nur so kann die nötige Offenheit entstehen, damit der Supervisor seine Inputs (Korrekturen, Anregungen usw.) ohne Zurückhaltung zur Verfügung stellt und der Therapeut für sich ungeniert selektioniert, welche er davon nutzen und in die Sitzung einbeziehen oder weglassen möchte.

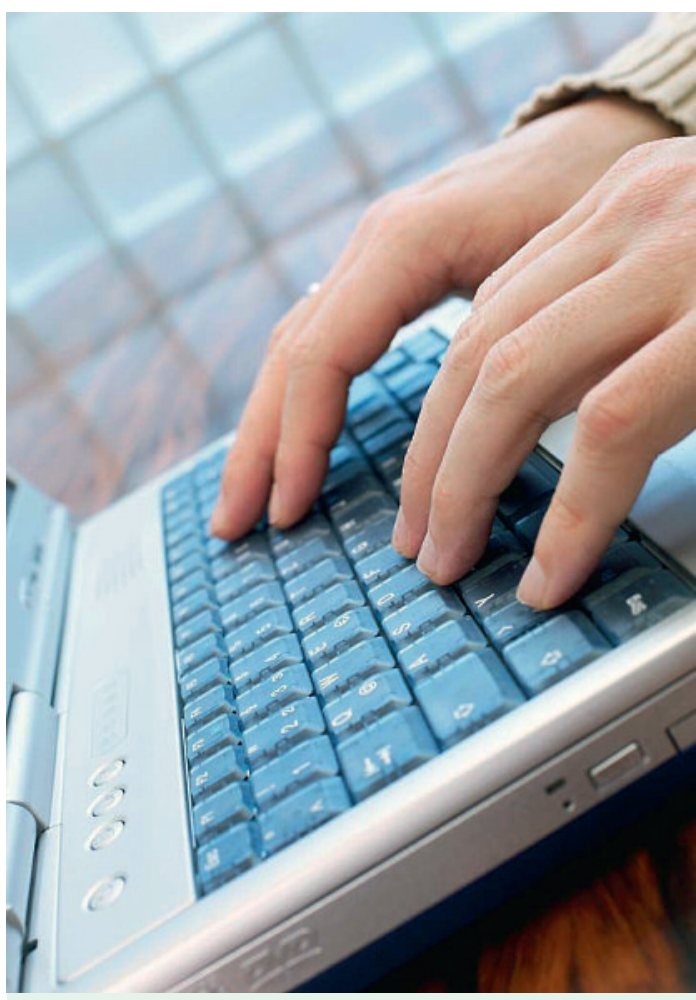

Der Supervisor schreibt seine Anmerkungen zum Therapieverlauf in ein Notebook mit zusätzlichem externen Bildschirm. Dieser steht beim Therapeuten, der entscheidet, ob er die Anregungen berücksichtigen will. audiovisuelle Übertragung der Sitzung zu einem externen Supervisor erinnert durchaus an endoskopische Untersuchungen oder chirurgische Eingriffe, bei denen über Optiken und Übertragung auf Bildschirme Einblicke in Innenräume des Patientenkörpers geschaffen werden. In der Therapiesitzung erfolgt die Beobachtung des «Operationsgebietes» (unmittelbare Therapie- und Beziehungsarbeit zwischen Arzt und Patient beziehungsweise dessen Seelenräumen) durch die Kamera mit Übertragung auf einen Monitor nach aussen zum Supervisor.

Die Arbeit des verantwortlichen Operateurs/ Therapeuten wird unterstützt durch den wachsam zur Seite stehenden und scharf beobachtenden Assistenten/Supervisor. Je höher dessen Fachkompetenz und Erfahrung sind, umso mehr werden seine Kommentare die Effizienz des Therapeuten steigern und den Therapieprozess konstruktiv fördern. Das gibt zusätzliche Sicherheit für Therapeut und Patient.

\section{Ökonomische Qualitätssicherung [4] mit Lerneffekt}

Der punktuelle Einsatz einer solchen Qualitätssicherungsmassnahme verlangt Offenheit nicht nur vom Patienten, sondern auch vom Therapeuten. Letztlich weist er aber gerade Engagement und Transparenz in Sachen Qualitätsförderung aus.

Unsere mittlerweile bereits mehrjährigen Erfahrungen mit dieser auf psychiatrisch-psychotherapeutischem Gebiet neuartigen Methode haben eindrucksvoll gezeigt [5], wie sehr Patienten und Therapeuten von derartigen Sitzungen profitieren. Es scheint, als wirke sich die gebündelte Kraft von Therapeut und Supervisor durch die Live-Kommentare direkt und markant auf den Patienten aus. Darüber hinaus lässt sich anhand der Aufzeichnung auch später noch ein Therapieprozess sehr detailliert und präzise analysieren, was einen hohen Lehr- und Lernwert für den jeweiligen Therapeuten und allenfalls auch weitere Fachkollegen hat.

Zusammenfassend kann festgehalten werden, dass es durch die Kamera-Übertragung und Live-Kommentierung gelingt, ohne wesentliche Störung an 
einem Therapieprozess teilzunehmen und sich von dessen Qualität ein deutliches, unmittelbares Bild $\mathrm{zu}$ machen. Es ist für einen externen Beobachter/ Supervisor dadurch möglich, direkt auf die Effizienz der Therapiearbeit einzuwirken. In Zukunft wird dieses Instrument daher nicht nur zur Qualitätssicherung der täglichen Arbeit, sondern von uns speziell auch im Ausbildungsbereich vermehrt eingesetzt werden.

\section{Benefit für Patient und Therapeut}

Die Befragung sowohl von Patienten als auch von Therapeuten (und gelegentlich im Hintergrund anwesenden Fachkollegen) stimmt zuversichtlich, dass beide Seiten von derartigen Therapiesitzungen mit Live-Monitoring aussergewöhnlich, das heisst mehr als in konventionellen Sitzungen ohne präsenten Supervisor, profitieren.

\section{Der Therapeut bleibt immer frei, Inputs des Supervisors einzubeziehen oder wegzulassen}

Der Gefahr von Störung oder Manipulation des Therapieprozesses durch aussenstehende Dritte stehen Autonomie und Eigenverantwortung des Therapeuten entgegen. Er ist jederzeit frei, auf Interventionen des Supervisors zu reagieren oder nicht. Unbedingt zu beachten ist dabei - und im bisher gepflegten Setting wurde dies bewusst so gewählt -, dass Therapeut und Supervisor sich nicht nur der Zusammenarbeit für den Patienten verpflichtet fühlen, sondern auch keine Konkurrenzsituation zwischen sich entstehen lassen.
Der Aufwand eines in regelmässigen Abständen durchgeführten Live-Monitorings lässt sich durch den Benefit auch für die ambulante Praxis rechtfertigen. Als fachlich reizvoll hat sich erwiesen, dass mehrere Kollegen gemeinsam mit einem externen Supervisor in deren Praxen abwechslungsweise im Turnus Live-Supervisionen durchführen lassen. Entscheidender als die methodische Ausrichtung sind dabei Fachkompetenz und Erfahrung des Supervisors und das Vertrauensverhältnis zwischen ihm und den Kollegen/Therapeuten.

Letztlich profitiert der Patient am meisten, da für ihn auf diese einfache Art kumuliertes Know-how im Therapieprozess zur Verfügung gestellt wird. Natürlich finden jedoch auch die effizientesten Qualitätssicherungsmassnahmen letztlich nur dann breite Anwendung, wenn sie nicht nur ideell, sondern auch finanziell und darüber hinaus zum Beispiel in Ausbildung und Trainings lohnend sind.

\section{Literatur}

1 Cassis I. Das Parlament zum Thema Qualität. Schweiz Ärztezeitung. 2010;91(41):1599.

2 Babics J et al. Anmerkungen zum Q-Monitoring der FMH. Schweiz Ärztezeitung. 2010;91(41):1616-17.

3 Lachenmeier H. Überlegungen zur Qualitätssicherung der psychotherapeutischen Arbeit mit neurotischen Patienten. Schweiz Ärztezeitung. 1996;77(6):223-7.

4 Oggier W. Wie und was messen Ökonomen im Mental-Health-Bereich? Schweiz Ärztezeitung. 2001;82(41): 2180-2.

5 Pfaundler S, Lachenmeier H. Experiences with directly written live-supervision. Präsentation am Scientific Meeting der Schweizerischen Gesellschaft für Intensive Dynamische Kurzpsychotherapie (29. November 2008). 